\title{
Computer-assisted hand-held dynamometer: low-cost instrument for muscle function assessment in rehabilitation medicine
}

\author{
J. Harlaar ${ }^{1}$ \\ M. E. Roebroeck ${ }^{1,2}$ \\ G. J. Lankhorst ${ }^{1}$ \\ 'Department of Rehabilitation Medicine, Free University Hospital, De Boelelaan 1117, \\ 1081 HV Amsterdam, The Netherlands \\ ${ }^{2}$ Netherlands, Institute of Primary Health Care (NIVEL), Utrecht, The Netherlands
}

\begin{abstract}
In rehabilitation medicine, muscle function is assessed during the physical examination of the patient. Although a simple hand-held instrument improves the assessment of static strength, it is rarely used in clinical practice, where dynamic measurements are preferred. A computer-assisted hand-held dynamometer (CAHNDY) has been developed that enables the clinician to measure dynamic muscle function in a standardised manner, using simple (i.e. portable and low-cost) apparatus. The CAHNDY comprises a force transducer and a movement transducer interfaced to a personal computer. In the study dynamic measurement protocols are used, based on a biomechanical analysis of daily activities. In this way, iso-functional profiles can be established, describing the kinematics of muscle function in its functional context. Using the iso-functional profiles as a basis for standardisation, the double feedback algorithm of the CAHNDY assists the tester in meeting this standard, during maximal muscle function testing. In a multi-centre trial, the CAHNDY is evaluated by physical therapists for knee extensor and flexor function. The CAHNDY enables iso-functional dynamic muscle function testing, although application is limited by the maximal strength of the tester. It is concluded that the CAHNDY is suitable for use in rehabilitation medicine.
\end{abstract}

Keywords-Biomechanics, Dynamometer iso-functional, Functional disability, Impairment, Muscle strength, Physical therapy, Rehabilitation medicine

Med. \& Biol. Eng. \& Comput., 1996, 34, 329-335

\section{Introduction}

THE ASSESSMENT of muscle function is frequently used in rehabilitation medicine, orthopaedic medicine, neurology and physical therapy for clinical decision-making. Muscle testing is an integral part of the physical examination of the patient and is performed by manually resisting the intended movement of the group of contracting muscles (KENDALL et al., 1971). By asking the patient to contract maximally, muscle function is assessed as its maximum strength under that specific test condition.

The grading of muscle function is based on the personal impression of the examining clinician, and rated on an ordinal scale, from 0 (no contraction) to 5 (normal) (KeNDALL et al., 1971). This system was introduced to grade the severity of poliomyelitis (LOVETT, 1916) and became the de facto standard after a memorandum from the British Medical

Correspondence should be addressed to J. Harlaar; email j.harlaar@azvu.nl.

First received 6 April 1995 and in final form 22 March 1996.

(C) IFMBE: 1996
Research Council in 1943 (MEDICAL RESEARCH COUNCIL, 1943). This rather ill-defined system has very low accuracy and sensitivity, especially in the range of interest (ROEBROECK, 1994, VAN DER PLOEG et al., 1984; AITKENS et al., 1989; BOHANNON, 1986). In addition the score is influenced by factors other than muscle strength (NichOLAS et al., 1978). Despite this, the system is used by the vast majority of clinicians.

Muscle function is directly measurable as the maximal net moment of force, which can objectively and quantitatively be expressed as a mechanical quantity. This was already realised in the 18th century (PEARN, 1978), and attempts have been made ever since to use instruments for the assessment of muscle function (SALTER, 1955; AMUNDSEN, 1990). Counterweights, spring balances, pressure systems and strain gauges, in a fixed set-up, were used to restrain the effects of the muscle force. Although simple to operate, these did not result in widespread clinical use.

A hand-held dynamometer, as first described by Beasley (BEASLEY, 1956), has the advantage that it can be used during the physical examination of the patient. Many portable types of hand-held pocket dynamometer have been described (EDWARDS and MCDONNEL, 1974; MARINO et al., 1982; HYDE et al., 1983; VAN DER PlOEG et al., 1984; BOHANNON, 
1986; STUBERG and METCALF, 1988). Although it is argued that a better assessment of muscle function can be made using this simple handy instrument, there has not yet been widespread clinical acceptance. Apparently, this type of static, i.e. isometric, strength measurement is not perceived as making a major contribution to clinical decision-making.

The introduction of isokinetic dynamometry (HISLOP and PERRINE, 1967) was heralded with excitement, because of the possibility of 'dynamic' testing of the musculature, as it was believed that it could be used to infer functional capacity (ROTHSTEIN et al., 1987). However, isokinetic limb movements do not occur during normal activities (ROTHSTEIN et al., 1987; HARLAAR, et al., 1993), and so there is little a priori reason for greater inferential use than isometric measurements. This view is supported by the current literature (KNAPIK et al., 1983; LANKHORST et al., 1985; ROTHSTEIN, 1985; WALLA et al., 1985; STAM, 1990; KRAMER et al., 1993). Furthermore, the cost of investment and the operational costs of an isokinetic device are high, restricting its wide-ranging accessibility.

Manual assessment of muscle function is performed under the specific conditions of the physical examination. Unfortunately, clinical textbooks (KENDALL et al., 1971; DANIELS and WORTINGHAM, 1972; CYRIAX, 1978; VLEEMING et al., 1984) do not all list the same conditions under which these tests should be performed (LAMB, 1985). Furthermore, assessment of muscle function in current clinical practice is carried out using a variety of test positions of the patient and manual handling by the tester (MEIJs et al., 1990). The test position (which affects the contribution of gravity as well as the length of the muscles), test duration and verbal encouragement directly affect the maximum strength that can be generated (WEBSTERS, 1982). Therefore, the absence of standard conditions of application reduces the reliability of an instrument that measures muscle function. This also applies for the use of isokinetic dynamometers (VAN WEES et al., 1993).

This study starts by analysing the role of assessment of muscle function in disability analysis, with regard to the process of clinical decision-making. On the basis of this analysis, an instrument is devised: a computer-assisted handheld dynamometer. Its design is intended to tackle the problems outlined above in instrumented muscle function assessment.

\section{Method}

\subsection{Assessment of muscle function in rehabilitation medicine}

The reasons for clinical strength assessment are manifold. Sometimes muscle function is used to monitor the stage of a disease using the neuromuscular function, e.g. Guillain-Barré syndrome (VAN DER PLOEG, 1992; WILES and KARNI, 1983; STAM and BINKHORST, 1989). Duchenne muscular dystrophy (SCOTT et al., 1982) or polyneuropathy (FILlYAW et al., 1987).

In the context of rehabilitation, however assessment of muscle strength is mostly used for problem identification. When a patient experiences a restriction of some kind of movement, this may be caused by a loss of strength of one or more muscle groups. The assessment of muscle function enables the evaluation of whether this 'tentative impairment' is present. Subsequently, when a period of training is prescribed to strengthen the muscle group, there is a need for evaluation. In all situations, the assessment of the muscle function should be interpreted with regard to a reference value. Some studies describe normal population values (VAN DER Ploeg et al., 1991; AMUNDSEN, 1990; BÄCKMAN, 1988). The results for the contra-lateral side of the patient can also be used as a reference value. However, from the functional perspective of muscle function, the only valid reference is the ability to perform that specific movement well. To assess muscle function in this context, we analysed some daily activities using their biomechanical description, i.e. their kinetics and kinematics (HARLAAR et al., 1993).

The results for the knee (Fig. 1) show that there are two main characteristics relevant to the functional context of muscle function.
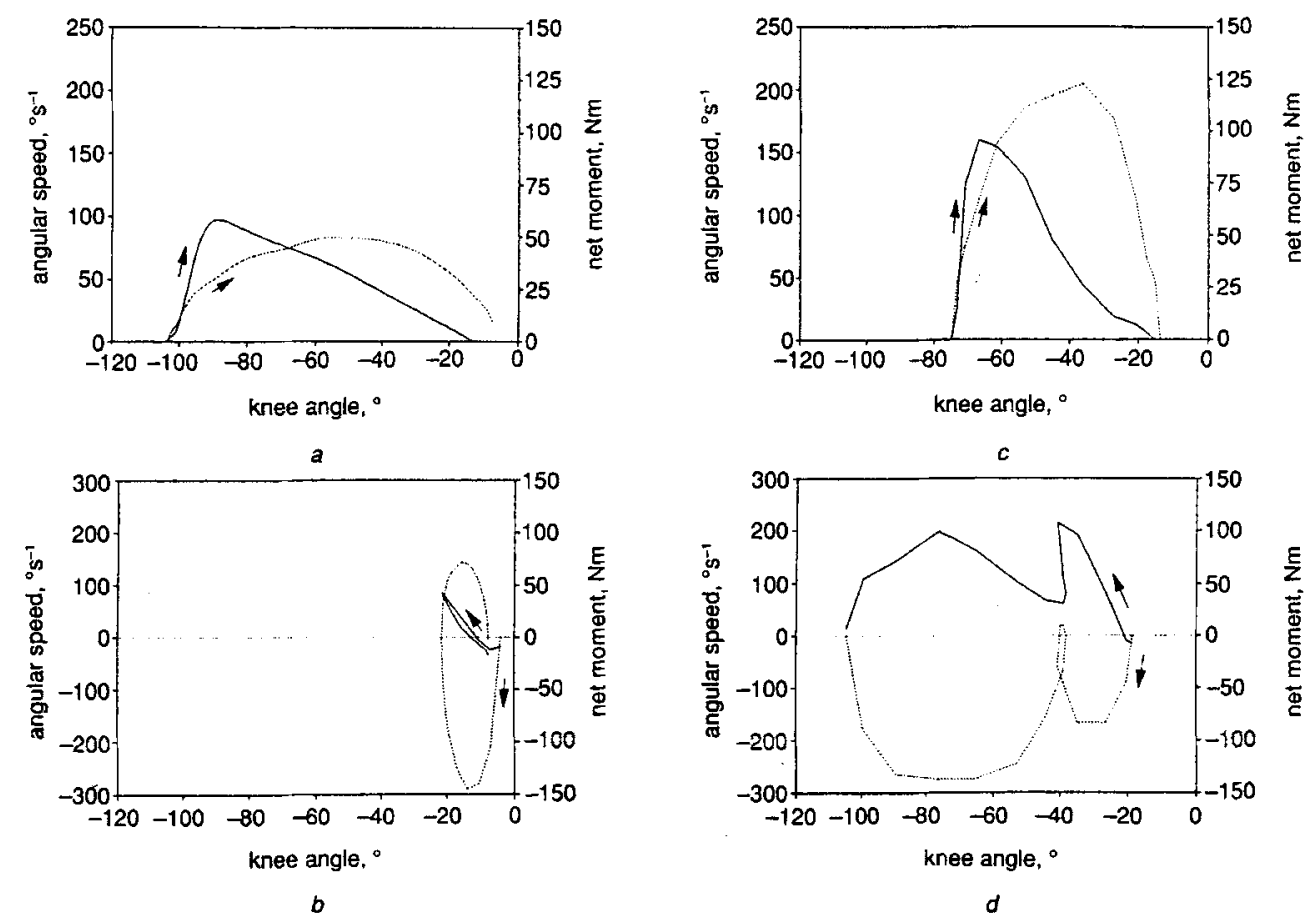

Fig. 1 Biomechanical description of knee function in its functional context: (a) rising from a chair; (b) level walking; (c) ascending stairs: (d) descending stairs; dashed lines $=$ angular speed; solid lines $=$ net moment; both are a function of knee angle: extension $=$ positive; full extension $=0^{\circ}$; moment values are adjusted for a body mass of $70 \mathrm{~kg}$ (reprinted from HARLAAR et al. (1993).) 
(a) The angular velocity around the joint builds up gradually, reaches its maximum halfway along the range of motion and then decreases again gradually to zero. This topology resembles a quadratic curve and is called isofunctional.

(b) For concentric muscle function, the maximum net moment is reached during the initial phase of the movement. For eccentric muscle function, the maximum net moment is reached at the end of the movement.

It is clear that neither characteristic applies to isokinetic testing, where, by definition, the angular speed is constant over the whole range of motion. In addition, in isokinetics, the course of the net moment of force follows the length-tension relationship of the muscle, with its maximum at the angle corresponding to the optimal muscle length. Instead, the maximum moment in the functional context is determined by the kinetics of the specific activity, which could serve as the reference value for muscle function assessment.

\subsection{Instrumentation}

The hardware of the CAHNDY consists of transducers, electronics and a (portable) computer (Fig. 2). The force between the hand of the clinician and the distal extremity of the patient is measured by a strain gauge-based force transducer*, cushioned on either side to fit both the hand of the clinician and the extremity of the patient. The force transducer has a range of $\pm 570 \mathrm{~N}$, with an accuracy of $0.5 \mathrm{~N}$. Assuming that the clinician applies the transducer perpendicular to the lever arm within $10^{\circ}$, the cross-talk from non-normal forces stays within $0 \cdot 2 \%$.

The electrogoniometer $\dagger$, used to measure the joint angle, is based on long strain gauges along a flexible cord and needs no alignment with the axis of rotation of the joint. Instead, both ends of the cord are aligned with the longitudinal axis of the joint's mutual limb segments. A foot-switch can be used to trigger the beginning and end of the measurement. The switch and both transducers are connected to custom-made electronics for signal processing. This includes amplifying the lowimpedance signals and limiting the bandwidth to $5 \mathrm{~Hz}$. The analogue signals are then converted to digital values with a sample frequency of $20 \mathrm{~Hz}$. These values, together with some status signals, are transmitted over a serial line (RS232) to the computer§, where a custom-made software program written in Turbo Pascal runs under the MS-DOS operating system. Fig. 3 shows the information flow diagram of the total system.

*HBM-Darmstad, Germany, type EF7A

† Penny \& Giles, Gwent, UK, type M180

$\$$ based on an Intel 386 processor and a VGA graphics display
Once the operator has activated a specific muscle function measurement protocol, the software program obtains the signals from the RS232 interface and displays them in real time on the screen, preferably as an XY plot of the net moment versus angle. The signals are concurrently evaluated against the protocol constraints to provide online feedback signals to the clinician and patient. When the measurement (which takes up to about $10 \mathrm{~s}$ ) is finished, the signal is frozen on the screen and is finally evaluated against the protocol-specific constraints, providing off-line feedback about the status of the measurement. A typical result is shown in Fig. 4.

\subsection{Muscle function measurement protocols}

Using the CAHNDY, dynamic measurements of muscle function can be performed. As proposed above, the measurement of muscle function should be considered in its functional context. This implies that every measurement should be specific to an activity. In the following, we use the function of the knee extensor muscle related to the activity 'rising from a chair' as an example. The two characteristics of muscle function derived from the embedding in the functional context are translated into constraints and a reference. The constraints are set by the isofunctional profile, meaning that the knee extensor muscle function test should be performed using the range of motion and speed profile identical to those found in rising from a chair (ROEBROECK et al., 1994). The reference is the net moment value that has to be overcome by the strength of the knee extensors to perform the task of rising from a chair normally. In other words, the reference establishes a value that is conditional to good performance of that specific movement. Such a reference might serve as a clinical goal.

As it is rather impractical for the clinician to mimic precisely the complete kinematics of the functional ability, we define a target as a crucial point in the total movement. For 'rising from a chair' (Fig. la), the target is defined as $90^{\circ}$ flexion, where the contraction speed is $50^{\circ} \mathrm{s}^{-1}$ (ROEBROECK et al., 1994). While the patient is asked to contract maximally, the clinician controls the movement by restraining the effort of the patient in such a way that the constraint of the test is met, moving at $50^{\circ} \mathrm{s}^{-1}$ at $90^{\circ}$ of flexion, expressed as target:50@90. This ensures a standard performance of the test.

Two feedback loops are implemented (Fig. 3) to assist the clinician in performing according to the standard. First, when the angle of interest (i.e. $90^{\circ}$ of flexion) is reached within $5^{\circ}$, an acoustic feedback is generated. A metronome signal is also beating. Secondly, after the measurement, the signals are evaluated against the defined target:50@90 through the use of a special algorithm. In the case of incorrect performance (i.e. not within $20 \%$ of the target), the clinician is corrected with a 'feedback signal' in natural language, e.g. 'the target

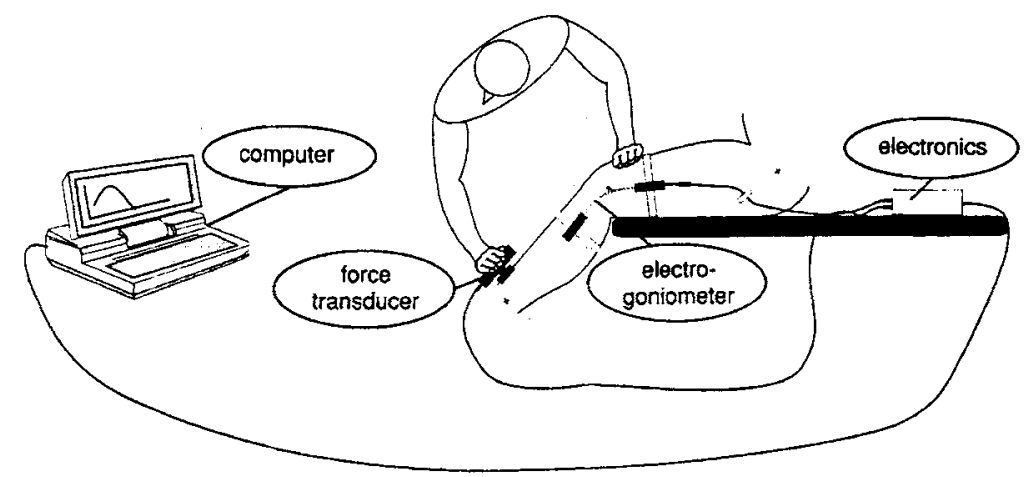

Fig. 2 Overview of CAHNDY instrumentation and its use in a knee function test 


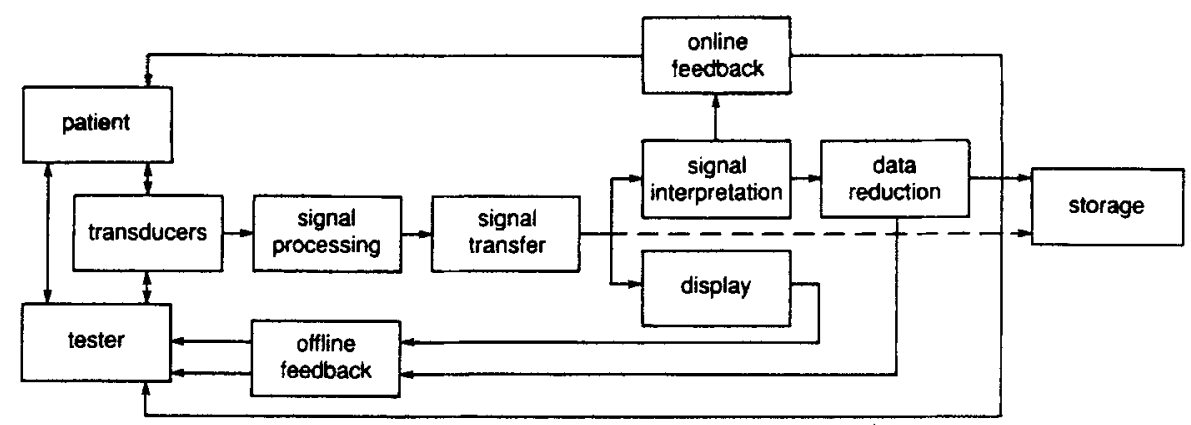

Fig. 3 Information flow diagram of CAHNDY

velocity was reached too eariy' or 'velocity was far too low.' This off-line feedback is accompanied by the information from the frozen signals on the screen. In addition to these feedback loops, the experience of the clinician, after initial training with the CAHNDY, is also expected to contribute to a standard performance.

A typical measurement session starts with the physical examination of the patient, during which specific bony points are marked on the skin. For a knee muscular function test, these are the hip joint (trochanter major), the knee joint (lateral epicondyle) and the ankle joint (apex of the lateral malleolus). The point of application of the force transducer is also marked, which is at $80 \%$ of the tibia length for the knee function test.

Before the actual measurement, some individual data of the patient are entered, comprising the weight of the patient and the lever arm of the force transducer, in addition to identification data. After the electro-goniometer has been attached to the joint (Fig. 2), it is calibrated using a large adjustable goniometer. When the clinician has selected the appropriate protocol, the graphical feedback screen is activated. After instruction to the patient, the clinician starts and stops the actual measurements using the foot-switch. When at least two measurements of correct performance (i.e. according to the protocol-specific constraints) have been made, the clinician has the option to close this protocol or continue for a higher result.

\section{Evaluation}

The clinical applicability of the CAHNDY was evaluated in a multi-centre trial. At five physical therapy locations (two therapists per location), 58 patients with strength impairment around the knee were measured in three sessions per patient. The design of the first part of this study was aimed at quantification of test-retest reliability, as well as inter-clinician reliability for isometric knee extensor strength. These results have been reported extensively elsewhere (ROEBROECK, 1994). It was concluded that the error between sessions, expressed as the standard error of measurement (SEM) (ROEBROECK et al., 1993), typically equals $10 \mathrm{Nm}$, either as the intra-observer or inter-observer reliability.

In the same trial, dynamic measurements were performed to evaluate the feasibility of the dynamic standardisation, using the 'rising from a chair' protocol (ROEBROECK, 1994). The physical therapists all underwent two short training sessions before the study. After the study, they judged the practicability of the CAHNDY to achieve a standard dynamic performance of the new test as fair to good, although rather complex. It was seen as a challenge to their professional skill to perform correctly. The feedback control in the CAHNDY to guide them to a correct performance of the measurements was especially appreciated.

Apparently, they were quite successful, as in only four patients the measurements were not performed correctly. In

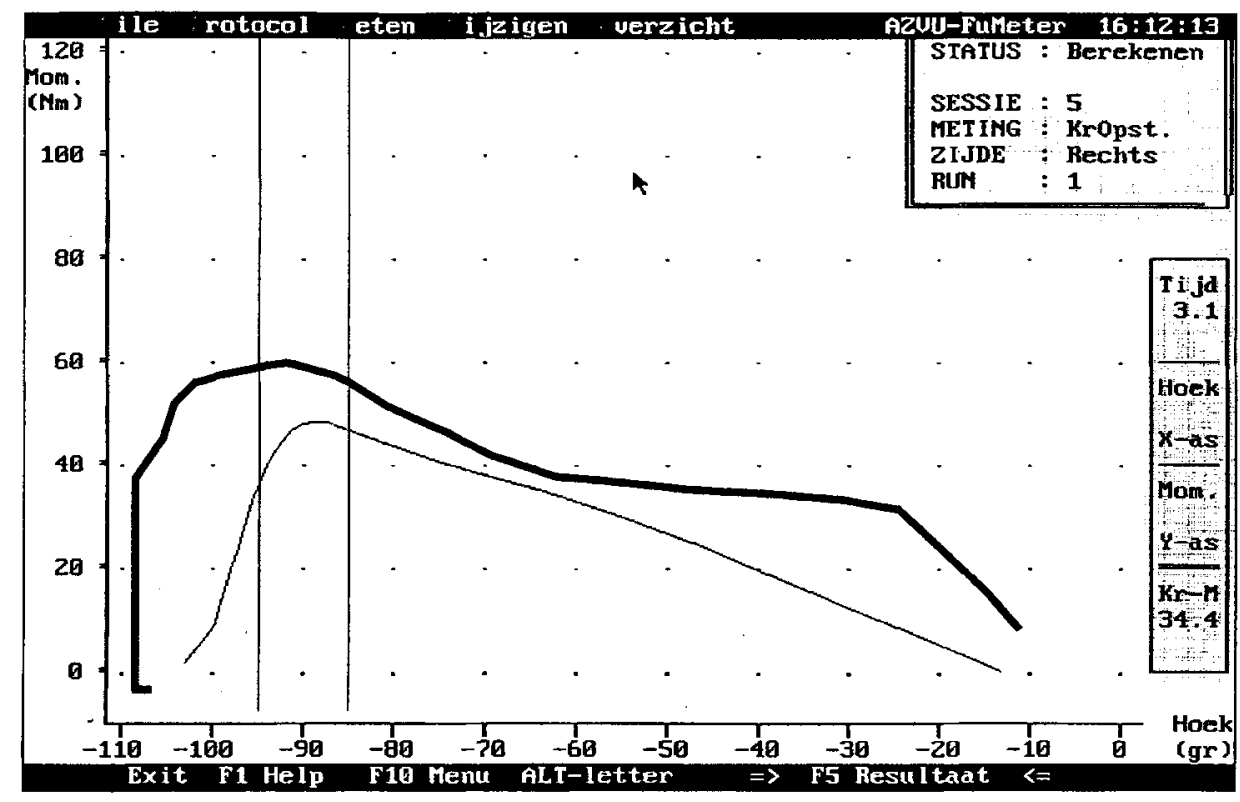

Fig. 4 Typical iso-functional measurement of asymptomatic subject with the CAHNDY, using 'rising from chair' reference and 50@90 target; knee angle is on the abscissa; negative values refer to degrees of flexion; the net moment of knee extension is plotted as the ordinate; bold line =trajectory of the actual measurement; the subject-specific reference curve is shown as thin line 
these cases, it was mentioned that the patients had greatly insufficient muscle function to perform the test, even at minimal resistance. On the other hand, some patients were too strong for the therapist, and so the therapist could not control the contraction.

\section{Discussion}

\subsection{Indeterminacy}

A given movement requires a particular external moment envelope to accompany an appropriate velocity envelope. Thus, it is clear that the function of a muscle is not a quantity per se, but only has a meaning in relation to its context. In clinical practice, it is important to notice that muscle function is conditional to functional activities, with each specific ability imposing a characteristic load on the muscle. Biomechanical analysis is a way of describing these characteristic loads in quantitative terms that can be directly related to isofunctional constraints and to a reference, when muscle function needs to be assessed outside the context of the ability, i.e. in an isolated test.

There are two problems with the isolated testing of muscle function. The first problem is linked to the indeterminacy problem, i.e. the net moment around a joint cannot be related to the force of just one muscle group. Muscle function serves the functional ability, as a part of the co-ordinative pattern of muscle activation, which might imply a significant amount of co-contraction (VAN INGEN SCHENAU et al., 1992; DOORENBOSCH et al., 1994). This means that inference of muscle function from a net joint moment establishes the lower limit of the reference value. Consequently, realistic reference values might be higher than those deducted from biomechanical analysis.

The second problem is that, in a strength test, it is necessary to elicit maximal effort from the patient. The willingness to do so markedly affects the result, and there is no simple objective method of distinguishing between the physiological and the psychological end points of effort (SALTER 1955). Furthermore, even if maximal voluntary effort is plausible, a nonvoluntary inhibition of the activation of the muscle might be present, especially at lower speeds (i.e. higher net moments) (PERRINe and EdGergton, 1978; BOBbert and HARLAAR, 1992; GÜLCH, 1994).

A third concern to be raised is the level of antagonist contraction during a strength test, which might obscure the real potential of the agonist muscle function. Fortunately, the amount of co-contraction is usually low, although in pathological cases simultaneous EMG measurements might be necessary to assess this assumption (BARATTA et al., 1988; SNOW et al., 1993).

Altogether, the relationship between the results of isolated muscle function tests and functional performance is not straightforward. Experimental work, measuring both functional abilities and muscle function, should improve our knowledge of this relationship. For the time being, we feel that a step forward can be made with our approach to derive muscle function assessment protocols from a biomechanical analysis of activities. This is a better guarantee of a meaningful interpretation of muscle function assessment than isometric or isokinetic measurements.

\subsection{Practicability}

The advantage of hand-held instruments for muscle function assessment over methods using stationary equipment is that they can be integrated with the physical examination of the patient. Moreover, as an objective quantification of muscle function the results refer to the same conditions as during the physical examination. However, there are two major limitations to the use of hand-held devices that objectively quantify the amount of force.

First, the assessment of muscular strength as part of the physical examination of the patient is restricted by the limited strength of the examiner. For many muscle groups this is not likely to be a problem (MARINO et al., 1982; WIKHOLM and BohanNON, 1991; VAN DER PlOEG et al., 1991), and in children it is not a problem at all (BÄCKMAN, 1988; STUBERG and METCALF, 1988). For stronger muscle groups (e.g. the hip and knee extensors, the ankle plantar flexors) however, insufficient strength in the examiner might be a serious limitation. Fortunately, the functional reference levels are usually not higher than the maximal strength of the examiner, even for assessment of the knee extensor muscles (ROEBROECK, 1994).

Therefore, this limitation will usually not imply restrictions for valid conclusions, when the muscle function assessment is directed at daily activities. However, when the assessment is directed as maximal performance as in sports medicine, handheld assessment is insufficient. For that purpose, stationary devices, either isometric (AMUNDSEN, 1990) or isokinetic (KANNUS, 1994), are necessary.

\subsection{Standardisation}

Another concern with hand-held dynamometry is that, in order to obtain reliable results, it is very important to measure under standardised conditions. Although this holds equally for stationary devices (LAMB, 1985), the versatility and flexibility inherent in a hand-held instrument make this method more susceptible to errors originating from non-standardised testing. As isometric conditions are easy to standardise, hand-held testing has been limited to isometric testing (EDWARDS and MCDONNEL, 1974; MARINo et al., 1982; HYDE et al., 1983; VAN DER Ploeg et al., 1984; BOHANNON, 1986; STUBERG and METCALF, 1988; WIKHOLM and BOHANNON 1991). Standardisation of dynamic measurements has been achieved using isokinetic measurements (HISLOP and PERRINE, 1967, KANNUS, 1994).

This study has shown that dynamic standardisation can also be achieved in hand-held dynamometry, using computer assistance. To do so, the examiners were constrained to hit a target, defined as the crucial point of muscle function in its functional context. The selection of this point can be argued from a theoretical point of view. For the activity 'rising from a chair,' the target is defined as the point where the knee extensor muscles are loaded with the highest net moment. Alternatively, it might be argued that the point where the highest net power during the activity is achieved is crucial for completion of the activity. However, for the activity 'rising from a chair,' the base-line of the net moment is mainly determined by the quasi-static load imposed by the weight of the upper body (ROEBROECK et al., 1994). This load is relatively independent of variations in the low speed that is used to rise from the chair. For higher rising speeds, the net moment also increases (PAI and ROGERS, 1990), whereas the peak power increases twice as much.

\section{Conclusions}

Using computer assistance, it is feasible to perform handheld measurements of dynamic muscle function in a standardised way and in the functional context of daily activities. 
Acknowledgments-This work was supported by a grant from the Dutch Executive Agency for Technology Policy (StiPT).

The authors would like to thank Jos de Kreek, Luut Voor in ' $t$ holt, Rob Schuckman and Ronald Redmeijer for their contributions in engineering the CAHNDY.

\section{References}

AITKENS, S., LORD, J., BerNAUER, E., FoWler, W. M., LiebermaN, J. S., and BERCK, P. (1989): 'Relationship of manual muscle testing to objective strength measurements,' Muscle Nerve, 12, pp. 173177

AMUNDSEN, L. R. (Ed.) (1990): 'Muscle strength tesing. Instrumented and non-instrumented systems' (Churchill Livingstone, New York, USA)

BÄCKMAN, E. (1988): 'Methods of measurement of muscle function. Methodological aspects, reference values for children, and clinical applications,' Scand. J. Rehabil. Med., 20, Suppl. 20

Baratta, R., Solomonov, M., Zhou, B. H., Letson, D., ChUINARD, R., and D'ABRoSIA, R. (1988): 'Muscular coactivation. The rolw of the antagonist musculature in maintaining knee stability,' Am. J. Sports Med., 16, (2), pp. 113-122

BEASLEY, W. C. (1956): 'Influence of method on estimates of normal knee extensor force among normal and postpolio children,' Phys. Ther. Rev., 36, pp. 21-41

BOBBERT, M. F., and HARLAAR, J. (1992): 'Evaluation of momentangle curves in isokinetic knee-extension,' Med. Sci. Sports Exerc., 25, (2), pp. 251-259

BOHANNON, R. W. (1986): 'Manual muscle test scores and dynamometer test scores of knee extension strength,' Arch. Phys. Med. Rehabil., 66, pp. 390-392

CYRIAX, J. (1978): 'Textbook of orthopaedic medicine. Vol. 1, Diagnosis of soft tissue lesions' (Bailliere Tindall, London, UK) 7 th edn.

DANiEls, L., and Wortingham, C. (1972): 'Muscle testing: techniques of manual examination' (WB Saunders Company, London, UK) 3rd edn.

DoORENBOSCH, C. A. M., HarlaAR, J., Roebroeck, M. E., and LANKHORST, G. J. (1994): 'Two strategies of transferring from sitto-stand; the activation of biarticular muscles,' J. Biomech., 27, (11), pp. 1299-1307

EDwARDS, R. H. T., and MCDONNEL, M. (1974): 'Hand-held dynamometer for evaluating voluntary muscle function,' Lancet, ii, pp. $757-758$

FILLYAW, M. J., TANDAN, R., and BRADLEY, W. G. (1987): 'Serial evaluation of neuromuscular function in management of chronic inflammatory demyelinating polyneuropathy,'Phys. Ther., 67, pp. 1708-1711

GÜLCH, R. W. (1994): 'Force-velocity relations in human skeleton muscle,' Int. J. Sports Med., 15, Suppl. 1, pp. s2-s 10

HARLAAR, J., ROEBROECK, M. E., and LANKHORST, G. J. (1993): 'Quantitative assessment of joint function in clinical practice-a biomechanical based rationale for interpretation' in METRAL, $S$. (Ed.): Abstracts of the XIVth Congress of the International Society of Biomechanics, Paris, France, pp. 552-553

HISLOP, H. J., and PERRINE, J. J. (1967): 'The isokinetic concept of exercise,' Phys. Ther, 47, pp. 114-117

HYDE, S. A. SCOTT, O. M., and GODDARD, C. (1983): 'The myometer: the development of a clinical tool,' Physiotherapy, 69, pp. 424-427

KANNUS, P. (1994): 'Isokinetic evaluation of muscular performance: Implications for muscle testing and rehabilitation,' Int. J. Sports Med., 15, Suppl. 1, pp. s11-s18

KENDALL, H. O., KenDALl, F. P., and WADSWORTH, G. E. (1971): 'Muscles testing and function' (Williams and Wilkins Company, Baltimore, USA) 2nd edn.

KNAPIK, J. J. WRIGHT, J. E., MAWDSLEY, R. H., and BRAUN, J. M. (1983): 'Isokinetic, isometric and isotonic strength relationships,' Arch. Phys. Med. Rehabil., 64, pp. 77-80

KRAMER, J., NUSCA, D., FOWLER, P., and WebSTER-BOGAERT, S. (1993): 'Knee flexor and extensor strength during concentric and eccentric muscle actions after anterior cruciate ligament reconstruction using the semitendinosus tendon and ligament augmentation device,' Am. J. Sports Med., 21 (2), pp. 285-291
LAMB, R. L. (1985): 'Manual muscle testing' in RoTHSTEIN, J. M. (Ed.): 'Measurement in physical therapy' (Churchill Livingstone, New York, USA) pp. 47-56

LANKHORST, G. J., VAN DE STADT, R. J., and J. K. KORST, (1985): 'The relationships of functional capacity, pain, and isometric and isokinetic torque in osteoarthrosis of the knee,' Scand. J. Rehabil. Med., 17, pp. 167-172

LOVETT, R. W. (1916): 'The treatment of infantile paralysis' (Blakiston's Son \& Co., Philadelphia, USA)

Marino, M., Nicholas, J. A. Gleim, G. W., RosentHal, P., and NichoLAS, S. J. (1982): 'The efficacy of manual assessment of muscle strength using a new device,' Am. J. Sports Med., 10, pp. 360-364

MEDICAL RESEARCH COUNCIL (1943): 'Aids to the investigation of peripheral nerve injuries.' War Memorandum 7 (2nd edn.) (HMSO, London, UK)

MeiJs, P. J. M., Roebroeck, M. E., and HarlaAR, J. (1990): 'Assessment of strength and range of motion about the knee,' Ned. T. Fysioth., 100, pp. 258-266 (in Dutch)

Nicholas J. A., Sagega, A., Kraus, H., and Webb, J. N. (1978): 'Factors influencing manual muscle tests in physical therapy' $J$. Bone Joint Surg., 60-A, pp. 186-190

PAI, Y.-C., and Rogers, M. W. (1990): 'Control of body mass transfer as a function of speed of ascent in sit-to-stand,' Med, Sci. Sports Exer., 22, pp. 378-384

PEARN, J. (1978): 'Two early dynamometers: an history account of the earliest measurements to study human muscular strength, ' $J$. Neurol. Sci., 37, pp. 127-134

PERRINE, J. J., and EDGERTON, V. R. (1978): 'Muscle force-velocity and power-velocity relationships under isokinetic loading,' Med. Sci. Sports Exer., 10, pp. 159-166

ROEBROECK, M. E. (1994): 'Clinical assessment of muscle function with a computer-assisted handheld dynamometer. Ph.D. Thesis, Free University, Amsterdam, The Netherlands

RoEbroecK, M. E., HARLAAR, J., and LANKHORST, G. J. (1993): 'The application of generalizability theory to reliability assessment: an illustration using isometric force measurements,' Phys. Ther., 73, pp. 386-401

Roebroeck, M. E., Doorenbosch, C. A. M., HarlaAR, J., JaCobs, R., and LANKHORST, G. J. (1994): 'Biomechanics and muscular activity during sit-to-stand transfer,' Clin. Biomech., 9, pp. 235-244 ROTHSTEIN, J. M. (Ed.) (1985): 'Measurement in physical therapy' (Churchill Livingstone, New York, USA)

ROTHSTEIN, J. M., LAMB, R. L., and MAYHEW, T. P. (1987): 'Clinical uses of isokinetic measurements. Critical issues;, Phys. Ther., 67 (12), pp. 1840-1844

SALTER, N. (1955): 'Methods of measurement of muscle and joint function,' J. Bone Joint Surg., 37-B (3), pp. 474-491

SCOTT, O. M., HYDE, S. A. GODDARD, C., and DUBOWITZ, V. (1982): 'Quantification of muscle function in children: a prospective study in Duchenne muscular dystrophy,' Muscle Nerve, 5, pp. 291-301

SNOW, J. C., COOPERS, J., QUANBURY, A. O., and ANDERSON, J. E. (1993): 'Antagonist cocontraction of knee flexors during constant velocity muscle shortening and lengthening,' J. Electromyogr. Kinesiol., 3, pp. 78-86

STAM, H. J., and BINKHORST, R. A. (1989): 'Muscle strength impairment and disability in Guillain-Barre patients: a pilot study,' J. Rehabil. Sci., 2, pp. 108-110

STAM, H. J. (1990): Dynamometry of the knee extensors; isometric and isokinetic testing in healthy subjects and patients.' $\mathrm{PhD}$. Thesis. Erasmus University, Rotterdam, The Netherlands

STUBERG, W. A., and METCALF, W. K. (1988): 'Reliability of quantitative muscle testing in healthy children and in children with Duchenne muscular dystrophy using a hand-held dynamometer,' Phys. Ther., 68, pp. 977-982

van Ingen Schenau, G. J., Boots, G. J., De Groot, P. J. M., SNACKERS, R. J., and VAN WOENSEL, W. W. L. M. (1992): 'The constrained control of force and position in multi-joint movements,' Neurosci., 46, pp. 197-207

VAN DER PlOEG, R. J. O., OOSTERHUIS, H. J. G. H., and ReUVEKAMP, J. (1984): 'Measuring muscle strength,' $J$. Neurol., 231, pp. 200203

VAN DER Ploeg, R. J. O., Fidler, V., and Oosterhuis, H. J. G. H. (1991): 'Hand-held myometry: reference values,' J. Neurol. Nenrosurg. Psych., 54, pp. 244-247 
VAN DER PlOEG, R. J. O. (1992): 'Hand-held dynamometry.' PhD Thesis, Rijksuniversiteit Groningen, Groningen, The Netherlands VAN DER WEES, PH. J., BOBBERT, M. F., and HARLAAR, J. (1993): 'Use of isokinetic equipment in the Netherlands: an inventory', Geneeskunde en Sport, 26, pp. 197-202 (in Dutch)

VleEming, A., WINKel, D., and Meijer, O. G. (1984) 'Weke delen aandoeningen van het bewegingsapparaat. Deel 1, Anatomie in Vivo' (Bohn, Scheltoma \& Holkoma, Utrecht, The Netherlands) (in Dutch) 4th edn.

Walla, D. J., Albricht, J. P., McAuley, E., Martin, R. K., Eldridge, V., and EL-KhOURY, G. (1985): 'Hamstring control and the unstable anterior cruciate ligament-deficient knee', $A m$. J. Sports Med., 13(1), pp. 34-39.

WESTERS, B. M. (1982): 'Factors influencing strength testing and exercise prescription,' Physiotherapy, 68, pp. 42-44

WIKHOLM, J. B., and BOHANNON, R. W. (1991): 'Hand-held dynamometer measurements: tester strength makes a difference,' $J$. Orthop. Sports Phys. Ther., 13, pp. 191-198

WILES, C. M., and KARNI, Y. (1983): 'The measurement of muscle strength in patients with peripheral neuromuscular disorders,' $J$. Neurol. Neurosurg. Psych., 46, pp. 1006-1013

\section{Author's biography}

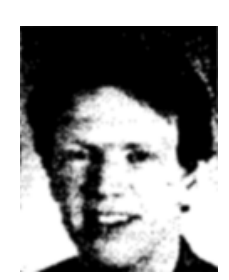

Jaap Harlaar obtained his MSc(Eng) in Electrical Engineering from the University of Technology, Twente, Enschede, The Netherlands, in 1985. He then joined the Department of Rehabilitation Medicine at the Free University Hospital, Amsterdam, where he established laboratories for human movement analysis and dynamometry. His work covers patient care, clinical trials, fundamental research, as well as biomechanically-based instrumentation design, all in the area of clinical biomechanics. His main research interest are the quantitative use of surface EMG to study co-ordination, clinical biomechanics and the methodology of technological developments inspired by a clinical problem. 